\title{
Growth and survival of lactic acid bacteria isolated from byproduct of virgin coconut oil as probiotic candidate for poultry.
}

\begin{abstract}
The main objective of this research is to investigate the potential of LABs isolated from processing byproduct of the VCO in in vitro conditions for use as probiotics in poultry. Forty eight LABs were isolated and four of them have been selected for further study i.e. Lh1, Lh2, Lh3 and Lh4. A series of tests carried out by studying the ability of bacteria to survive at 37 and $42^{\circ} \mathrm{C}$, tolerance of $\mathrm{LAB}$ at $\mathrm{pH} 2,0,5,7$ and 7,0 and tolerance to gastric juice as well as sensitivity to several antibiotics commonly was given to poultry. The survival of LABs was evaluated after 15, 30, 60, 90 and 120 and $300 \mathrm{~min}$ of incubation. The sensitivity test to antibiotics was performed by Muller Hinton's agar. All the bacteria showed tolerance and ability to grow at $\mathrm{pH} 5$ and 7, but only $\mathrm{Lh} 4$ enabled to tolerate at $\mathrm{pH} 2$. All of LAB can grow at gastric juice stimulated. Lh4 was not sensitive to all antibiotics (clear zones: $0.33 \mathrm{~mm}$ ) but the other LABs were sensitive (clear zones: 5-12 mm). The conclusion of this research is the ability of LABs to grow in in vitro conditions varies. The Lh4 has demonstrated its ability to grow and the best survival with the OD $(\lambda=580)$ is 1.99 after 300 min of incubation at $\mathrm{pH} 2$ and has shown the most resistant to all antibiotics tested with a wide clear zone $0.33 \mathrm{~mm}$, hence potentially be used for probiotic in poultry.
\end{abstract}

Keyword: Lactic acid bacteria; Byproduct VCO; Survival; Growth; Probiotic. 